\title{
Ex vivo evaluation of antifibrotic compounds in skin scarring: EGCG and silencing of PAI-1 independently inhibit growth and induce keloid shrinkage
}

Farhatullah Syed ${ }^{1,2,6}$, Rania A Bagabir ${ }^{1,2,6}$, Ralf Paus ${ }^{2,3}$ and Ardeshir Bayat ${ }^{1,2,4,5}$

Keloid disease $(K D)$ is a common fibroproliferative disorder of unknown etiopathogenesis. Its unique occurrence in human skin and lack of animal models pose challenges for KD research. The lack of a suitable model in KD and overreliance on cell culture has hampered the progress in developing new treatments. Therefore, we evaluated the effect of two promising candidate antifibrotic therapies: ( - )-epigallocatechin-3-gallate (EGCG) and plasminogen activator inhibitor-1 (PAl-1) silencing in a long-term human keloid organ culture (OC). Four millimeters of air-liquid interface (ALI) keloid explants on collagen gel matrix in serum-free medium ( $n=8$ cases) were treated with different modalities (EGCG treatment; PAI-1 knockdown by short interfering RNA (siRNA) and application of dexamethasone (DEX) as control). Normal skin $(n=6)$ was used as control (only for Do keloid-untreated comparison). Besides routine histology and quantitative (immuno-) histomorphometry, the key phenotypic and growth parameters of KD were assessed. Results demonstrated that EGCG reduced keloid volume significantly ( $40 \%$ by week 4 ), increased apoptosis ( $\geq 40 \%$ from weeks 1 to 4), and decreased proliferation ( $\leq 17 \%$ in week 2). EGCG induced epidermal shrinkage, reduced collagen-I and - III at mRNA and protein levels, depleted $98 \%$ of keloid-associated mast cells, and reduced the percentage of both cellularity and blood vessel count by week 4 . Knockdown of PAl-1 significantly reduced keloid volume by $28 \%$ in week 4 , respectively, and reduced collagen-I and -III at both mRNA and protein levels. As expected, DEX increased keloid apoptosis, decreased keloid proliferation, and collagen synthesis, but induced connective tissue growth factor overexpression. In conclusion, using keloid OC model, we provide the first functional evidence for testing candidate antifibrotic compounds in KD. We show that EGCG and PAI-1 silencing effectively inhibits growth and induces shrinkage of human keloid tissue in situ. Therefore, the application of EGCG, PAI-1 silencing, and other emerging compounds tested using this model may provide effective treatment and potentially aid in the prevention of recurrence of KD following surgery.

Laboratory Investigation (2013) 93, 946-960; doi:10.1038/labinvest.2013.82; published online 8 July 2013

KEYWORDS: dexamethasone; EGCG; immunohistochemistry; keloid; organ culture; PAI-1; siRNA

Keloid disease $(\mathrm{KD})$ is a benign hyperproliferative dermal disease of unknown etiopathogenesis with ill-defined treatment, ${ }^{1}$ which is unique to humans. ${ }^{2} \mathrm{KD}$ can arise following an abnormal wound healing process in genetically susceptible individuals. ${ }^{2}$ The hallmark of KD is excessive deposition of extracellular matrix (ECM) in the dermis. ${ }^{3}$

The lack of an animal model that can truly mimic human $\mathrm{KD}$ in vivo condition imposes limitations when investigating functional activities of potential therapeutic agents in treating KD. ${ }^{4,5}$ However, these models have failed to provide the essential components of skin tissue that occur in vivo, such as mature ECM, blood vessels, inflammatory cells, and intrinsic and extrinsic biochemical interactions. Therefore, the ultimate aim of this study was to determine the applicability of recent research findings in existing in vitro keloid models to our recently developed keloid organ culture (OC) model. ${ }^{6}$

OC of human skin has already been extensively utilized as a useful method for investigating skin biology. ${ }^{7,8}$ Therefore, we reported the development of a serum-free ex vivo model of human KD, in which keloid tissue is organ cultured at the

\footnotetext{
${ }^{1}$ Plastic and Reconstructive Surgery Research, Manchester Institute of Biotechnology (MIB), University of Manchester, Manchester, UK; ${ }^{2}$ Institute of Inflammation and Repair, University of Manchester, Manchester, UK; ${ }^{3}$ Department of Dermatology, University of Lubeck, Lubeck, Germany; ${ }^{4}$ Department of Plastic and Reconstructive Surgery, University Hospital South Manchester NHS Foundation Trust, Wythenshawe Hospital, Manchester, UK and ${ }^{5}$ The University of Manchester, Manchester Academic Health Science Centre, Manchester, UK

Correspondence: Dr A Bayat, BSc (Hons), MB, BS, PhD, Plastic and Reconstructive Surgery Research, Manchester Institute of Biotechnology (MIB), University of Manchester, 131 Princess Street, Manchester M1 7DN, UK. E-mail: ardeshir.bayat@manchester.ac.uk

${ }^{6}$ The first two authors contributed equally to this work.

Received 23 November 2012; revised 14 May 2013; accepted 27 May 2013
} 
air-liquid interface (ALI). ${ }^{6}$ This keloid OC model has several advantages: first, keloid explants remain viable in culture for up to 6 weeks (without major deterioration of tissue morphology); second, the maintenance of keloid cellular and molecular microenvironments, which are capable of exerting their biochemical and molecular effects on the tissue, allow examination of KD pathophysiology in situ; third, antifibrotic agents and gene silencing using RNA interference (RNAi) technology can be used to understand the cellular and molecular interactions in both the epidermis and dermis that maintain keloid tissue growth; and, finally, novel candidate antikeloid agents can be preclinically tested in this OC system under clinically relevant ex vivo conditions. $^{6}$

Using recent advances in keloid ${ }^{9}$ and normal skin OC models, ${ }^{8}$ the keloid OC model permits the growth of $4 \mathrm{~mm}$ explants in a collagen matrix at the ALI in supplemented William's E (WE) medium for up to 6 weeks. ${ }^{6}$ In this model, dexamethasone (DEX) treatment serves as a robust positive control that inhibits keloid tissue growth. ${ }^{6}$ While currently, corticosteroids are the first-line pharmacological therapy for the management of $\mathrm{KD}$, there is no effective treatment identified to date that can completely eradicate as well as reduce recurrence of KD. ${ }^{1}$ Therefore, there is an urgent need for the development of effective, novel, antikeloid therapies that complement surgical excision of keloid tissue.

One promising new antikeloid agent, which awaits clinical evaluation, is ( - )-epigallocatechin-3-gallate (EGCG). ${ }^{4,10}$ This has been previously tested in human-derived keloid fibroblasts transplanted onto nude mice, and showed decreased collagen production and subsequent reduction of keloid. ${ }^{4,10}$ Reportedly, another important factor for excessive fibrogenesis in $\mathrm{KD}$ is the overexpression of plasminogen activator inhibitor-1 (PAI-1) in fibroblasts, which elevates collagen production. ${ }^{11}$ Knocking down PAI-1 using either short interfering RNA (siRNA) inhibited collagen synthesis in keloid fibroblasts. ${ }^{12}$

The aim of this study was to validate EGCG and PAI-1 silencing in an actual human keloid OC model to generate optimal, clinically relevant preclinical evidence whether or not these antikeloid strategies deserve further clinical testing. EGCG treatment and PAI-1 knockdown in situ were compared with DEX as a positive control. The results showed that EGCG treatment and PAI-1 silencing both effectively inhibit growth and induce shrinkage of human keloid tissue in situ.

\section{MATERIALS AND METHODS}

\section{Patient Recruitment and Preparation of Keloid OC}

Only patients presenting with features typical of KD, with no previous intralesional treatment, ${ }^{3,6}$ were recruited for this study. In addition, following routine therapeutic excision of keloid scars, each sample underwent independent additional histopathological confirmation of diagnosis of their keloid, which was performed by an experienced dermatopathologist.
All keloid patients recruited gave full informed consent in our ethically approved study. Eight keloid cases and six control subjects (normal skin in an individual with no personal or family history of KD) with an age range of 16-43 years, of multiple ethnicities, were included in this study (Supplementary Table S1). After surgery, excised keloid tissue were washed and soaked in Hank's buffer (Sigma-Aldrich, Dorset, UK) for $30 \mathrm{~min}$. Then, $4-\mathrm{mm}$ punch biopsies (explants or OC) were cut and embedded in collagen gel matrix on ALI and maintained with serum-free, supplemented WE medium (Sigma-Aldrich), as described previously ${ }^{6}$ (Figure 1a). Media with or without treatment were changed every third day.

\section{DEX and EGCG Administration}

DEX and EGCG (Sigma-Aldrich) were dissolved in dimethyl sulfoxide. Four millimeters of ALI keloid OCs were maintained in serum-free, supplemented WE media containing either $50 \mu \mathrm{g} / \mathrm{ml}$ DEX, as positive control, or $100 \mu \mathrm{g} / \mathrm{ml}$ EGCG up to 4 weeks. Dimethyl sulfoxide/WE-supplemented media were used as vehicle control. Media were changed every third day with fresh media with or without treatment. Tissue and media were collected at defined time points as described in Figure $1 b$.

\section{Predesigned PAI-1 siRNA for Knockdown of PAI-1 in the Keloid OC Model}

Predesigned PAI-1 siRNA was purchased from Ambion (Paisley, UK) Applied Biosystems (Warrington, UK) (Table 1). Keloid OC (4-mm) punch biopsies were transiently transfected with PAI-1 siRNA and scrambled siRNA (250 and $500 \mathrm{~nm}$ ) using siPORT NeoFX Transfection agent (Applied Biosystems) according to the manufacturer's instructions.

In one set of experiment, media were replaced every $72 \mathrm{~h}$ with fresh WE serum-free, supplemented media containing the same amount of siRNA, until 4 weeks. In the second set of experiment, after first transfection with PAI-1 siRNA, the media were replaced every $72 \mathrm{~h}$ with fresh media until 4 weeks, without siRNA, to analyze the persistence of PAI-1 siRNA. Tissue was collected at days 0,1 , and 3 , and weeks $1-4$ in RNAlater, formalin, and in the culture media for analysis Figure 1b.

\section{Transfection of PAI-1/Scrambled siRNA in Keloid OC Using siPORT NeoFX}

The protocol was followed according to the manufacturer's instructions. Briefly, for preparation of siRNA and reagent dilutions, a sterile tube (round or V-bottom) was used. When possible, master mix was prepared to minimize variability. siPORT NeoFX Transfection agent $(3 \mu \mathrm{l})$ was diluted in OptiMEM I medium $(50 \mu \mathrm{l})$ and incubated at room temperature for $10 \mathrm{~min}$. Then, PAI-1 (250 and $500 \mathrm{~nm}$ ) or Scrambled (250 and $500 \mathrm{~nm}$ ) siRNA were diluted into Opti-MEM I medium $(50 \mu \mathrm{l})$. Thereafter, the diluted siPORT NeoFX Transfection Agent and diluted siRNA were combined. Subsequently, this 

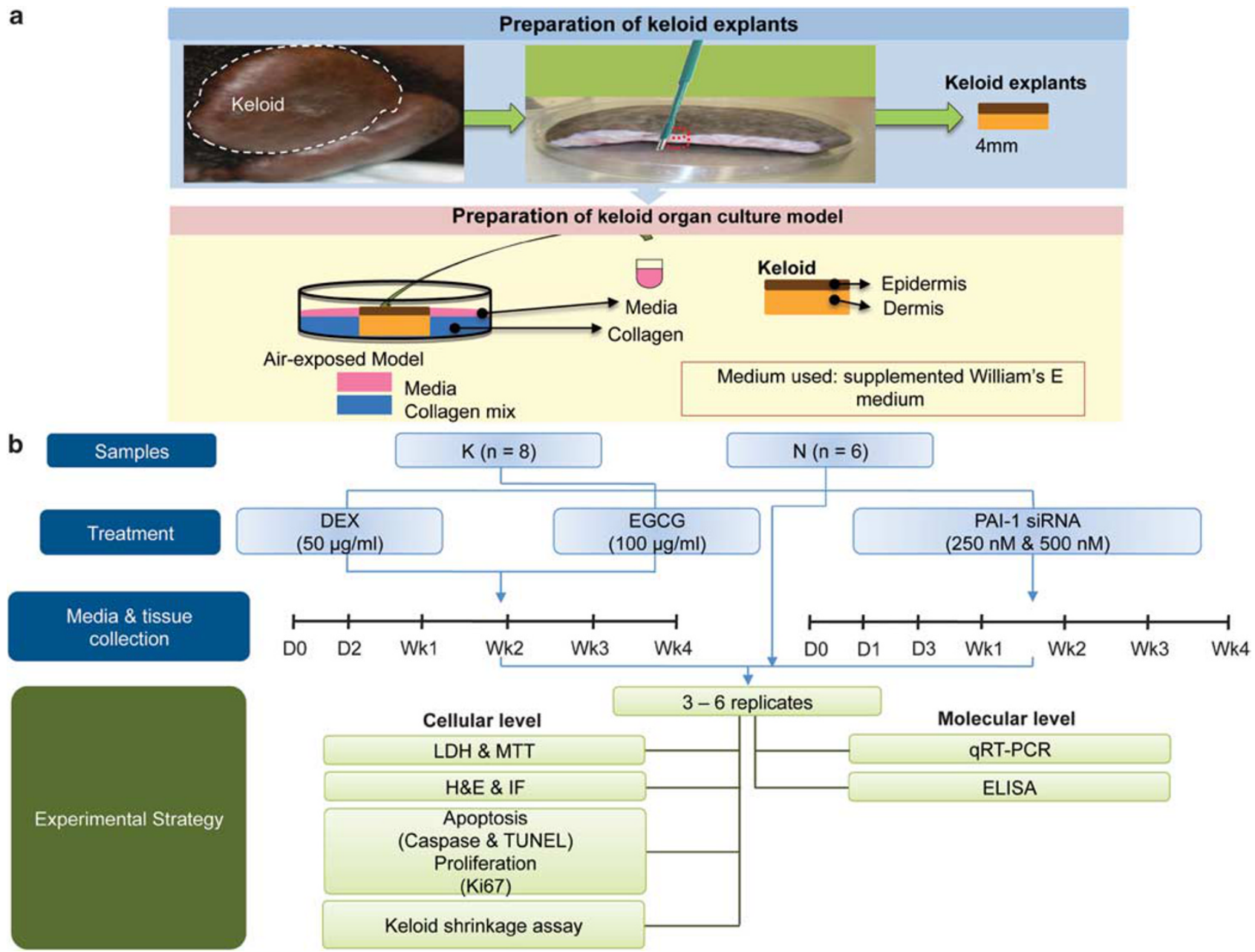

Figure 1 Experimental design for the functional evaluation of potential antifibrotic compounds in ex vivo keloid model. (a) Schematic representation of keloid ex vivo model setup. Harvested keloid tissue was dissected into $4 \mathrm{~mm}$ diameter using a standard punch biopsy kit. Explants were embedded in rat-tail collagen matrix (BD Bioscience, Oxford, UK) as air-liquid interface (ALI), also named air-exposed, in 24-well plates. (b) Different treatment regimen, predetermined timeline for media and tissue collection after treatments, and experimental strategy were used in this study. K: keloid tissue samples; N: normal skin samples; DEX: dexamethasone, EGCG: ( - )-epigallocatechin-3-gallate; PAI-1: plasminogen activator inhibitor-1;siRNA: short interfering RNA; nM: nanomolar; D: day; Wk: week; LDH: lactate dehydrogenase; MTT: methylthiazol tetrazolium; H\&E: hematoxylin and eosin; IF: immunofluorescence; TUNEL: TdT dUTP nick-end labeling; qRT-PCR: real-time quantitative reverse transcription-polymerase chain reaction; d ELISA: enzyme-linked immunosorbent assay.

was gently mixed by pipetting up and down or flicking the tube a few times, before it was incubated for $10 \mathrm{~min}$ at room temperature to allow transfection complexes to form. After incubation, siRNA/siPORT NeoFX Transfection Agent transfection complexes was dispensed into wells of the keloid OC containing $500 \mu \mathrm{l}$ of (final volume) media and the keloid OC culture plates were incubated at $37^{\circ} \mathrm{C} / 5 \% \mathrm{CO}_{2}$. The transfection was then repeated every $72 \mathrm{~h}$ in the keloid OC for up to 4 weeks.

\section{Cytotoxicity and Viability/Metabolic Activity Assays}

The cytotoxicity using lactate dehydrogenase (LDH) assay and viability/metabolic activity using methylthiazol tetrazolium (MTT) assay (Roche, West Sussex, UK) were
Table 1 PAI-1 siRNA information

\begin{tabular}{ll}
\hline siRNA ID & S10013 \\
\hline $\begin{array}{l}\text { Cat. no. } \\
\text { Gene symbol }\end{array}$ & $\begin{array}{l}4390824 \\
\text { SERPINE1 } \\
\text { Gene name }\end{array}$ \\
& $\begin{array}{l}\text { Serpin peptidase inhibitor, clade E (nexin, } \\
\text { plasminogen activator inhibitor type 1), member 1 } \\
\text { Gene aliases }\end{array}$ \\
NCBI location & Chr. 7: 100770379-100782547 \\
Chromosome & \\
UniGene ID & Hs.414795 \\
Species & Human \\
\hline
\end{tabular}



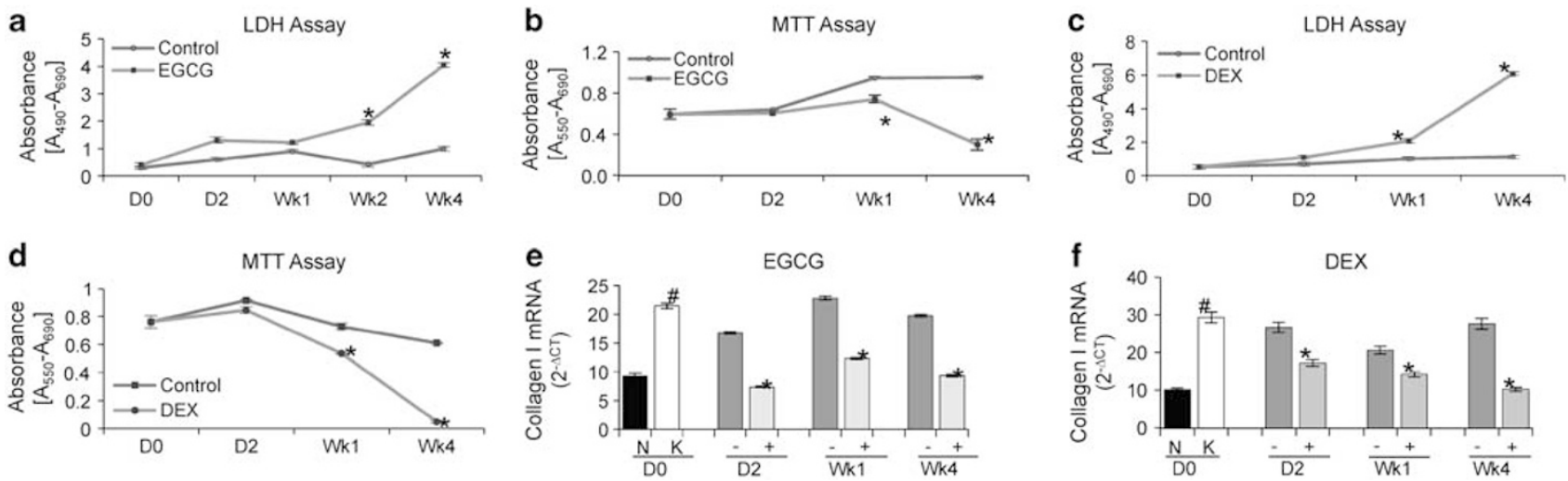

Figure 2 Effect of (-)-epigallocatechin-3-gallate (EGCG) and dexamethasone (DEX) treatment on keloid OC. (a) Cytotoxicity (lactate dehydrogenase $(\mathrm{LDH})$ assay) and (b) viability/metabolic activity (methylthiazol tetrazolium (MTT) assay) measurement in EGCG-treated keloid OC. ${ }^{*} P \leq 0.04$ significantly different compared with vehicle-treated control. (c and d) LDH and MTT measurement in DEX-treated keloid OC. ${ }^{*} P \leq 0.03$ significantly different relative to vehicle-treated control. (e) Comparison of collagen-I mRNA expression upon EGCG and (f) DEX treatments. ${ }^{~} P \leq 0.01$ significantly different relative to normal skin $(\mathrm{N})$ at day $0 .{ }^{*} P<0.03$ significantly different relative to vehicle-treated control. D: Day; Wk: week.

measured in the media and tissue, respectively, following the manufacturer's protocol. ${ }^{6}$

\section{Apoptosis and Ki-67 Detection In Situ}

Terminal dUTP nick-end labeling (DeadEnd TUNEL; Promega, Southampton, UK) was used as a marker for apoptosis combined with fluorescence immunohistochemistry of cell proliferation marker Ki-67 as described previously. ${ }^{6,13}$

\section{Histology}

Tissue sections were stained for morphological analysis with hematoxylin and eosin using standard methodology. ${ }^{3,6}$ Micrographs were captured using Olympus BX51 (Olympus, Essex, UK) microscope. For cellularity/inflammation and $\mathrm{CD} 31^{+}$cell analysis, percentage was calculated relative to day 0 in the dermis from four independent patients.

\section{Immunofluorescence}

A range of markers listed in Supplementary Table S2 were localized on paraffin sections $(5 \mu \mathrm{m})$. Micrographs for immunofluorescence (IF) were captured using confocal microscope (Zeiss LSM 510META, Hertfordshire, Germany). Quantitative immunohistomorphometry was carried out as described previously. ${ }^{14}$

\section{Collagen I and III ELISA}

Quantitative collagen I and III protein expression secreted in the media, collected from treated keloid OC and vehicletreated control, were measured using capture sandwich and indirect ELISA following a protocol that was optimized previously. ${ }^{3,6}$

\section{RNA Extraction, cDNA Synthesis, and qRT-PCR}

Keloid OC tissue biopsies were collected at different time points (Figure 1b) post-treatment in RNAlater (Applied Biosystems). RNA extraction was performed using RNeasy mini kit (Qiagen, Crawley, UK). ${ }^{15}$ Subsequently, $1 \mu \mathrm{g}$ mRNA was converted to cDNA using qScript cDNA Synthesis kit (Quanta BioSciences, Gaithersburg, MD, USA) ${ }^{15}$ Real-time quantitative reverse transcription polymerase chain reaction (qRT-PCR) was carried out with standard protocol ${ }^{15}$ using a number of gene-specific primers (Supplementary Table S3).

\section{Shrinkage of Keloid Volume, Epidermal Thickness, Reduced Cellularity, and Vascularity}

Keloid shrinkage assay, epidermal thickness shrinkage, cellularity, and vascularity were assessed by means of histology and immunohistochemistry as described previously. ${ }^{6}$

\section{Statistical Analysis}

Each experiment was performed independently for two to three times. Data were expressed as mean \pm standard error (s.e.m.). Statistical evaluation of data was performed using ANOVA in SPSS 16.0 software program (SPSS Inc., Chicago, IL, USA). Differences with $P<0.05$ were considered significant.

\section{RESULTS \\ EGCG is Cytotoxic and Inhibits Collagen Production in Keloid OC}

EGCG treatment stimulated cytotoxicity and significantly reduced $(P<0.03)$ viability/metabolic activity from week 1 to week 4 compared with the vehicle-treated (dimethyl sulfoxide) control (Figures $2 \mathrm{a}$ and $\mathrm{b}$ ). However, DEX induced higher cytotoxicity and lower viability/metabolic activity (Figures $2 \mathrm{c}$ and $\mathrm{d}$ ) compared with the EGCG-treated group. EGCG and DEX both significantly $(P<0.02)$ reduced collagen I (Figures $2 \mathrm{e}$ and $\mathrm{f}$ ) and collagen III (Figures $3 \mathrm{a}$ and $\mathrm{b}$ ) transcription relatively early (on day 2). At protein level, there was significant $(P<0.05)$ reduction of secreted collagen I (Figure 3c) at day 2 in EGCG-treated group, whereas there was a significant reduction in secreted collagen III $(P<0.05)$ 

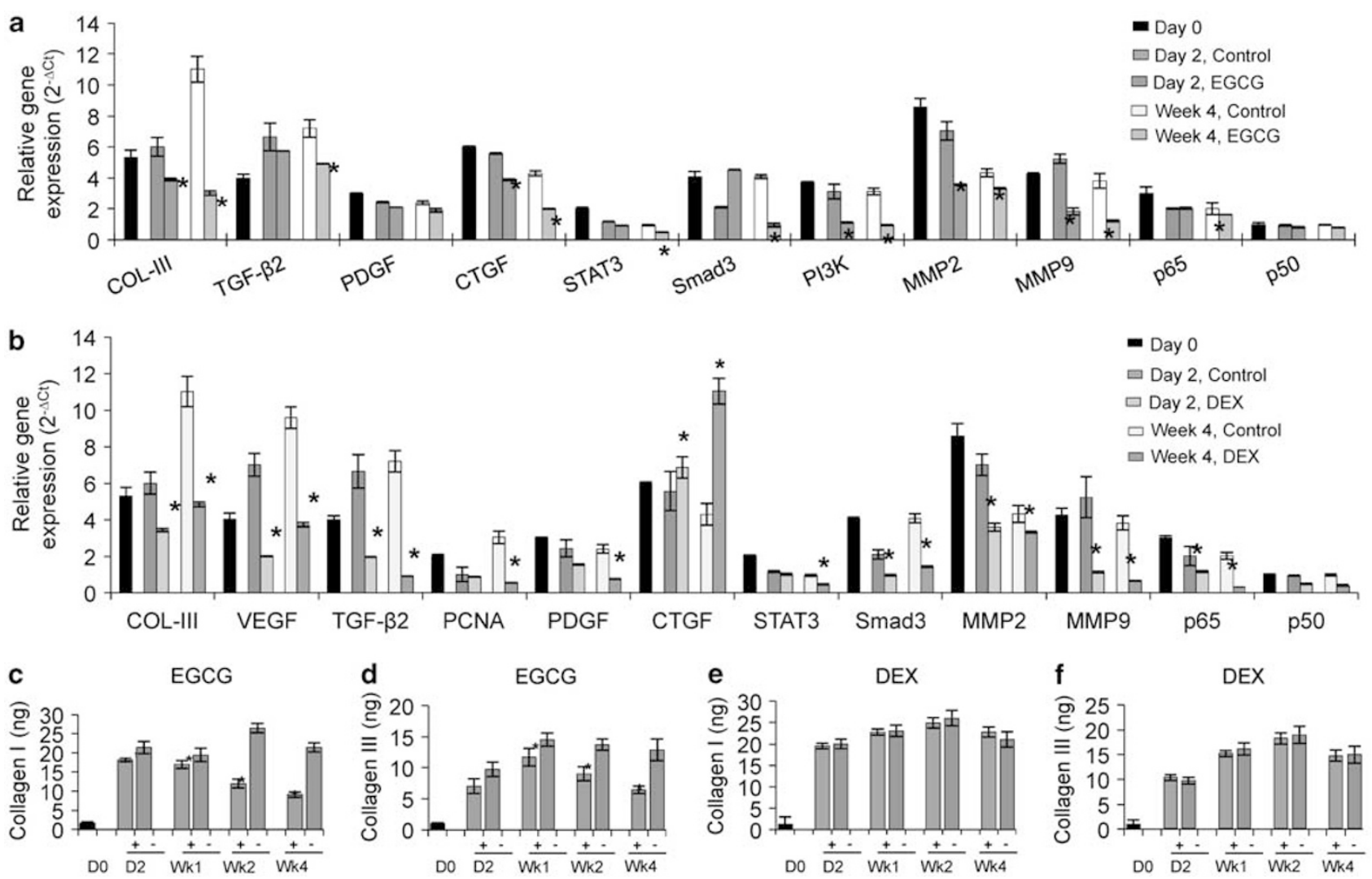

Figure 3 Relative gene and protein expression of profibrotic molecules after different treatments. (a) Relative gene expression of profibrotic molecules after ( - )-epigallocatechin-3-gallate (EGCG) treatment. (b) Relative gene expression of profibrotic molecules after dexamethasone (DEX) treatment. (c and d) Collagen I and III protein expression. ${ }^{*} P \leq 0.05$ comparing EGCG-treated with vehicle control. Bars represent standard error. (e and $\mathbf{f}$ ) Collagen I and III protein expression. Bars represent standard error. (Note: D0: day 0, indicates either keloid scar tissue or keloid scar tissue transported media collected after surgery, without any treatment before being organ cultured.) COL: collagen; TGF: tumor growth factor; PDGF: platelet-derived growth factor; STAT3: signal transducer and activator of transcription 3; PI-3K: phosphoinositol 3-kinase; MMP: matrix metalloproteinase; VEGF: vascular endothelial growth factor; PCNA: proliferating cell nuclear antigen; CTGF: connective tissue growth factor; D: Day; Wk: week.

at week 1 onwards (Figure 3d). Surprisingly, DEX showed no significant changes in the secreted collagen I and III at the protein level (Figures $3 \mathrm{e}$ and f). In contrast, collagen I immunoreactivity was also markedly decreased (Figure 4) in EGCG-treated OC. In contrast, overall, EGCG reduced intrakeloid collagen synthesis more efficiently than DEX.

\section{EGCG Downregulates Transcription of Major Fibrosis- Associated Pathways}

As shown in Figure 3a, transcription of the investigated fibrotic-associated genes (vascular endothelial growth factor $(V E G F)$, matrix metalloproteinases (MMP 2 and 9), and TGF- $\beta 2$ ), which have been implicated in KD pathobiology, ${ }^{16,17}$ were inhibited by EGCG treatment as measured by qRT-PCR (Figure 3a) compared with the vehicle-treated control. Similar effects on major fibrosis-associated pathways were also seen with DEX (Figure 3b). Interestingly, we also measured the expression profile of TGF- $\beta$ II at the protein level, in keloid OC, and the results showed visible reduction in TGF- $\beta$ II expression at week 1 and week 4 treatment with EGCG when compared with the control group (Figure 4a).

\section{DEX Upregulates CTGF Expression in Keloid OC, While EGCG Downregulates CTGF}

Connective tissue growth factor (CTGF) is a recognized key growth factor during wound healing and in KD pathobiology. ${ }^{18,19}$ Hence, the effect of EGCG and DEX on CTGF expression was also investigated. Interestingly, CTGF gene expression was significantly downregulated by EGCG treatment (Figure 3a). In contrast, there was a significant $(P \leq 0.01)$ overexpression of CTGF mRNA in DEX-treated keloid OC compared with the vehicle-treated control (Figure 3b), which also correlated with the in situ protein level (Figure 4b). Thus, glucocorticoid treatment can indeed enhance the in situ expression of a major keloid-promoting growth factor, while EGCG has the opposite effect.

\section{EGCG Depletes Mast Cells}

As shown in Figure 5a, mast cells are shown to be associated with keloid. In our keloid OC model, treatment with EGCG significantly decreased mast cell numbers $(P \leq 0.01)$, and a 93-98\% depletion of mast cells were observed by week 4 in comparison to the vehicle-treated control. 
DO anti-TGF- $\beta$ II+DAPI

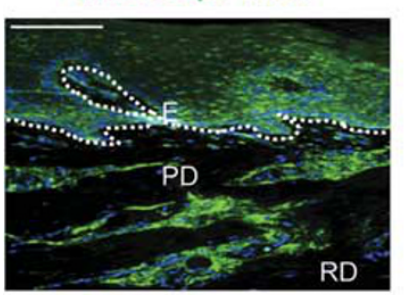
$\mathrm{RD}$

b

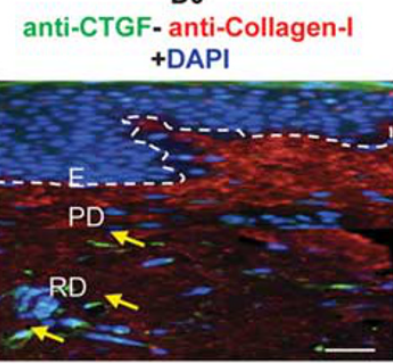

\section{임}
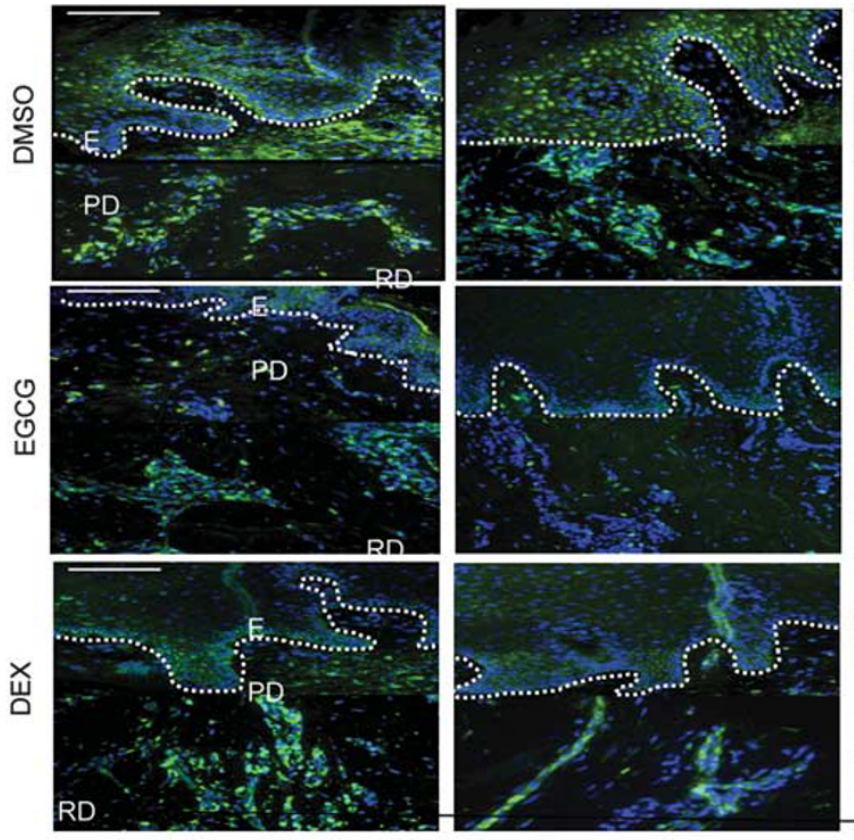

D3

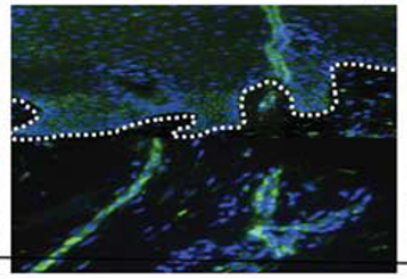

Wk1

anti-TGF- $\beta \|+D A P I$

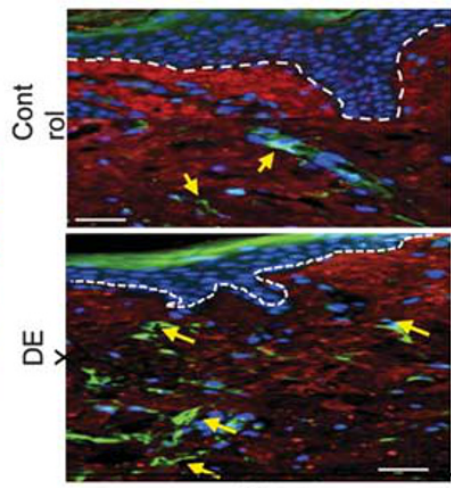

D2

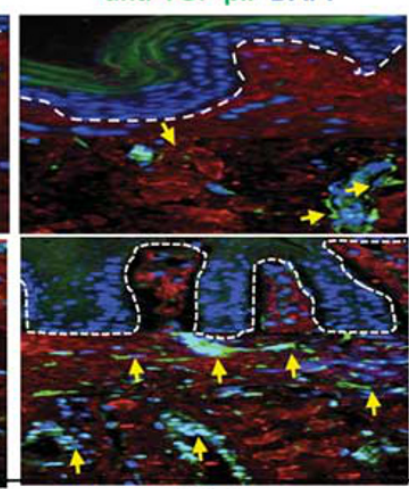

Wk1

anti-CTGF- anti-Collagen-I +DAPI
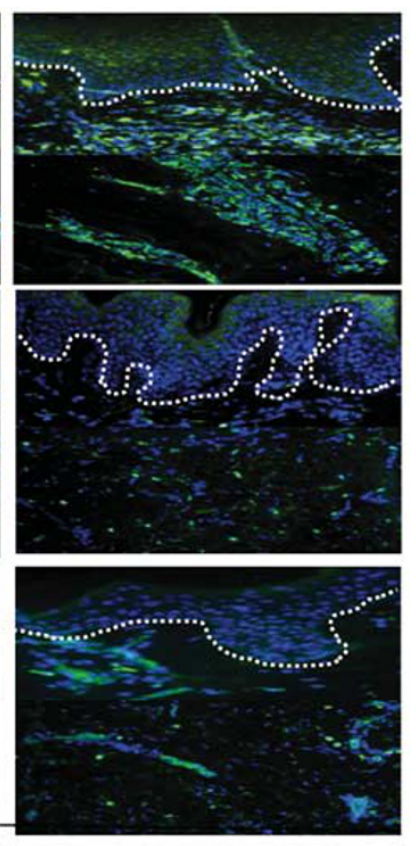

Wk4
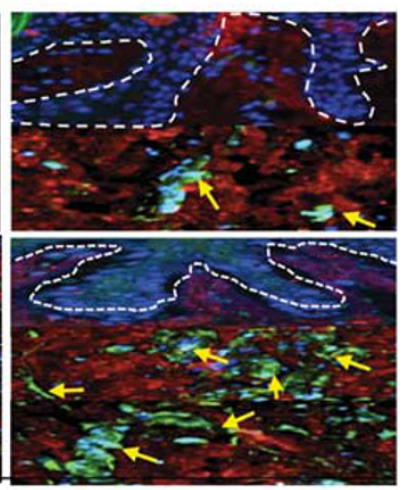

Wk4

Figure 4 Expression pattern of fibrotic markers after different treatments at protein level. (a) Effect of ( - )-epigallocatechin-3-gallate (EGCG) and dexamethasone (DEX) treatment on tumor growth factor (TGF)- $\beta$ Il expression profile in keloid organ culture (OC) at different time points. Scale bar: $\times 100$ magnification. (b) Effect of DEX on collagen I (red) and CTGF (green) expression using dual-color immunofluorescence staining in keloid OC. Scale bar: $\times 200$ magnification. DAPI: 4',6-diamidino-2-phenylindole; DMSO: dimethylsulfoxide; D: day; Wk: week; E: epidermis; PD: papillary dermis; RD: reticular dermis.

\section{EGCG Stimulates Intrakeloid Apoptosis and Inhibits Proliferation}

Reduction of mast cell numbers may reflect the pronounced level of apoptosis in keloid OC observed after EGCG treatment $(P \leq 0.03)$, starting at week 1 and persisting up to week 4 . The increased percentage of apoptotic cells compared with the vehicle control was evidenced by quantitative caspase- 9 , active caspase-3 (Figures $6 \mathrm{a}$ and b), and TUNEL immunohistomorphometry (Figure 6c). Furthermore, a sharp decline in proliferation $\left(\mathrm{Ki}-67^{+}\right.$cells) was detected in the EGCG-treated OC by week $4(P<0.05)$ compared with vehicle-treated controls (Figure 6c). These proapoptotic effects of EGCG were only slightly less impressive as those seen in the positive control, that is, DEX-treated keloid OC (Figures 6a-c).

\section{EGCG Induces Keloid Shrinkage in OC and Reduces Epidermal Thickness, Percentage of both Cellularity, and Blood Vessel Density}

To evaluate the therapeutic potential of EGCG in the treatment of KD, the weight of keloid OC was measured weekly for up to 4 weeks post-treatment. Results showed visible shrinkage of keloid weight reflected by significant $(P=0.01)$ reduction of keloid OC weight by $28 \%$ at week 3 and by $39 \%$ at week 4 compared with the vehicle-treated control (Figures $7 \mathrm{a}$ and $\mathrm{b})$. H\&E staining and quantitative CD31 analyses showed that EGCG reduced the percentage of both cellularity/inflammation and blood vessel $\left(\mathrm{CD} 31^{+}\right.$cells $)$immunoreactivity up to $46 \%$ and $273 \%$ at day 2, respectively, relative to day 0 (Figures $8 \mathrm{a}-\mathrm{d}$ ). Furthermore, there was a 
a

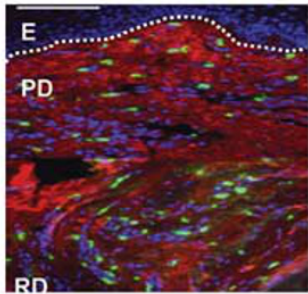

DO

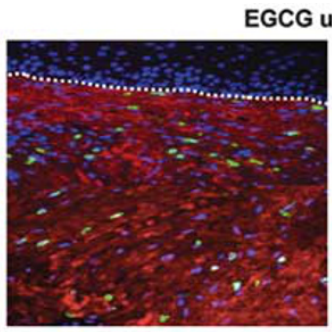

Wk1
EGCG untreated

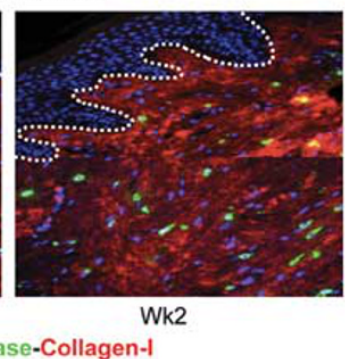

DAPI-Tryptase-Collagen-I

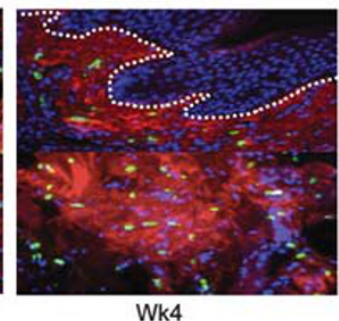

Wk4

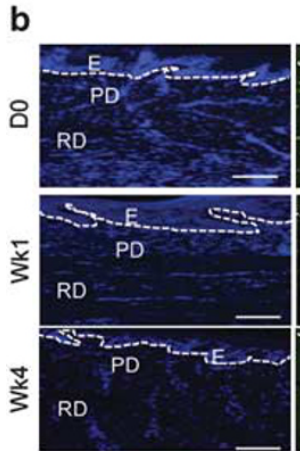

DAPI

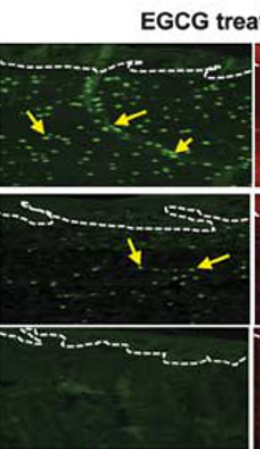

Tryptase

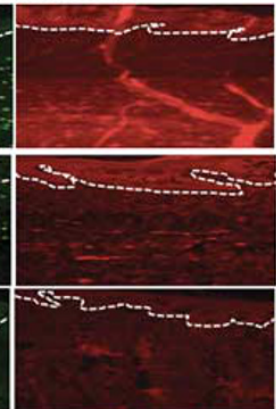

Collagen-1

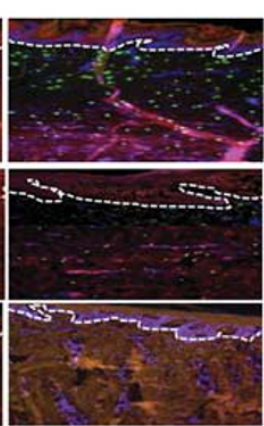

Merged
C

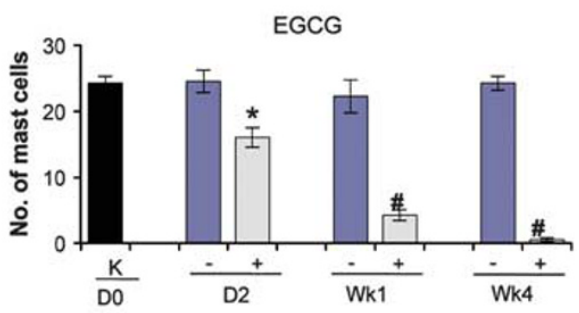

Figure 5 Effect of (-)-epigallocatechin-3-gallate (EGCG) on mast cell number in keloid organ culture (OC) model. (a) Immunohistochemistry analysis of mast cell (green) numbers and collagen 1 (red) expression pattern in keloid OC, without EGCG treatment at different time points. Scale bar: $\times 200$ magnification. (b) Effect of EGCG on collagen I (red) and mast cell (green) immunoreactivity using immunofluorescence assay. Scale bar: $\times 100$ magnification. Representative results from three independent experiments are shown in (a-c). Bars represent standard error. (Note: D0: day 0, indicates keloid scar tissue, collected after surgery, without any treatment before the start of OC.) (c) Immunomorphometric analysis of mast cell numbers with and without EGCG treatment generated from four random micrographs taken at $\times 200$ magnification. ${ }^{*} P<0.05$ and ${ }^{\#} P=0.01$ relative to vehicle-treated control. E: epidermis; PD: papillary dermis; RD: reticular dermis; DAPI: 4',6-diamidino-2-phenylindole; D: day; Wk: week.

significant $(P<0.05)$ reduction in the epidermal thickness upon EGCG treatment at week 2 (28\% reduction) reaching $59 \%$ reduction in week 4 , relative to the vehicle control group (Figure 8e). The reduction in cellularity/inflammation and vascularity percentage was significant $(P \leq 0.01)$ compared with the vehicle-treated control. Keloid size, cellularity/inflammatory, and blood vessels were also suppressed by DEX treatment as reported previously in keloid OC; ${ }^{6}$ however, it appears (data comparison between EGCG from this study and DEX-treated groups from the previously reported study ${ }^{6}$ ) that the effect of EGCG is more efficient than DEX.

\section{PAI-1 Silencing Reduces Keloid Volume and Collagen Synthesis}

Knockdown efficiency analysis revealed successful reduction of PAI-1 gene a day after transfection with an average reduction of $60 \%$ at $250 \mathrm{nM}$ PAI-1 siRNA concentration, as opposed to the average reduction of $10 \%$ using a $500 \mathrm{nM}$ concentration (Figure 9a). More importantly, PAI-1 siRNA gene silencing visibly suppressed the expression of PAI-1 protein in keloid OC from week 1 to week 4 (Figure 9b). This represents the first successful gene knockdown in an intact human keloid tissue in situ and provides proof-of-principle that gene silencing in the current keloid OC model.
After PAI-1 siRNA administration, a significant difference in cytotoxicity and viability/metabolic activity was observed up to week 4, with the $500 \mathrm{nM}$ compared with the vehicletreated control (scrambled oligos). However, $250 \mathrm{nM}$ siRNA induced a significant increase of cytotoxicity at week 2 , and only a slight change was observed with the viability/metabolic activity (at week 4) (Figures 10a and b).

The transcriptions of important PAI-1 downstream target genes, including collagen I at mRNA (Figure 10c), protein level (Figure 10d), and collagen III at mRNA level (Figure 11a), were effectively reduced a day post-transfection (250 nM siRNA) by an average of $54 \%$ and $30 \%$, respectively. By qRT-PCR, PAI-1 knockdown had no statistically significant $(P \geq 0.05)$ effect on the transcription of major profibrotic pathway players (Figure 11a). In addition, PAI-1 silencing significantly reduced collagen-I (Figure 11b) and III (Figure 11c) protein levels as well. This corresponded well to a noticeable decrease in the hyalinized collagen bundles in tissue sections (up to 4 weeks) in PAI-1-silenced keloid OC (Figure 11d). Interestingly, no major morphological differences in the tissue architecture were observed in the scrambled oligo-treated control keloid OC (Figure 11d). As PAI-1 siRNA transfection at the $250 \mathrm{nM}$ concentration was more effective than the $500 \mathrm{nM}$ concentration; hence, most of 


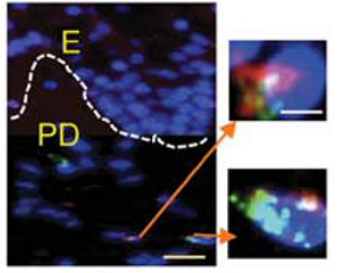

D0

Caspase-9

Caspase-3

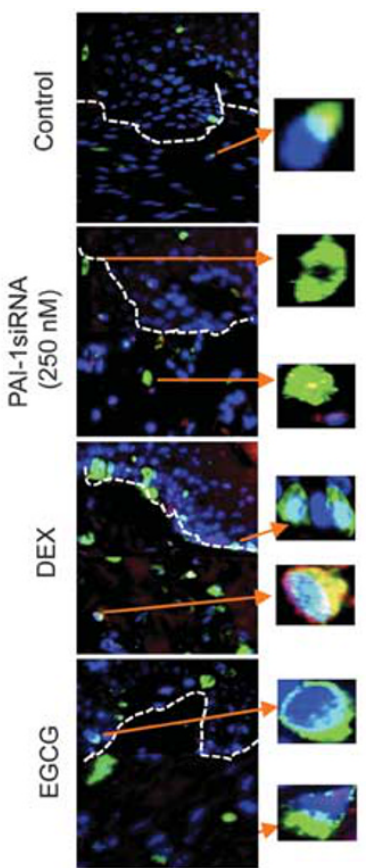

D2
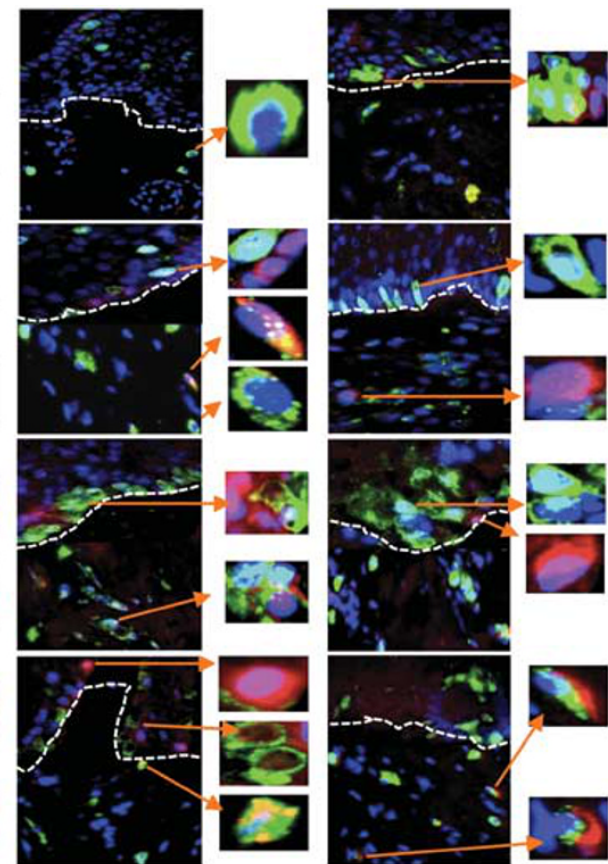

Wk1
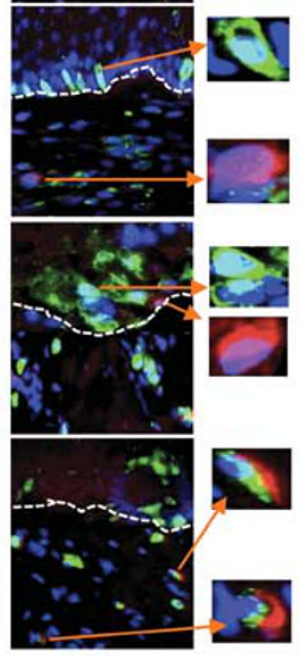

Wk4
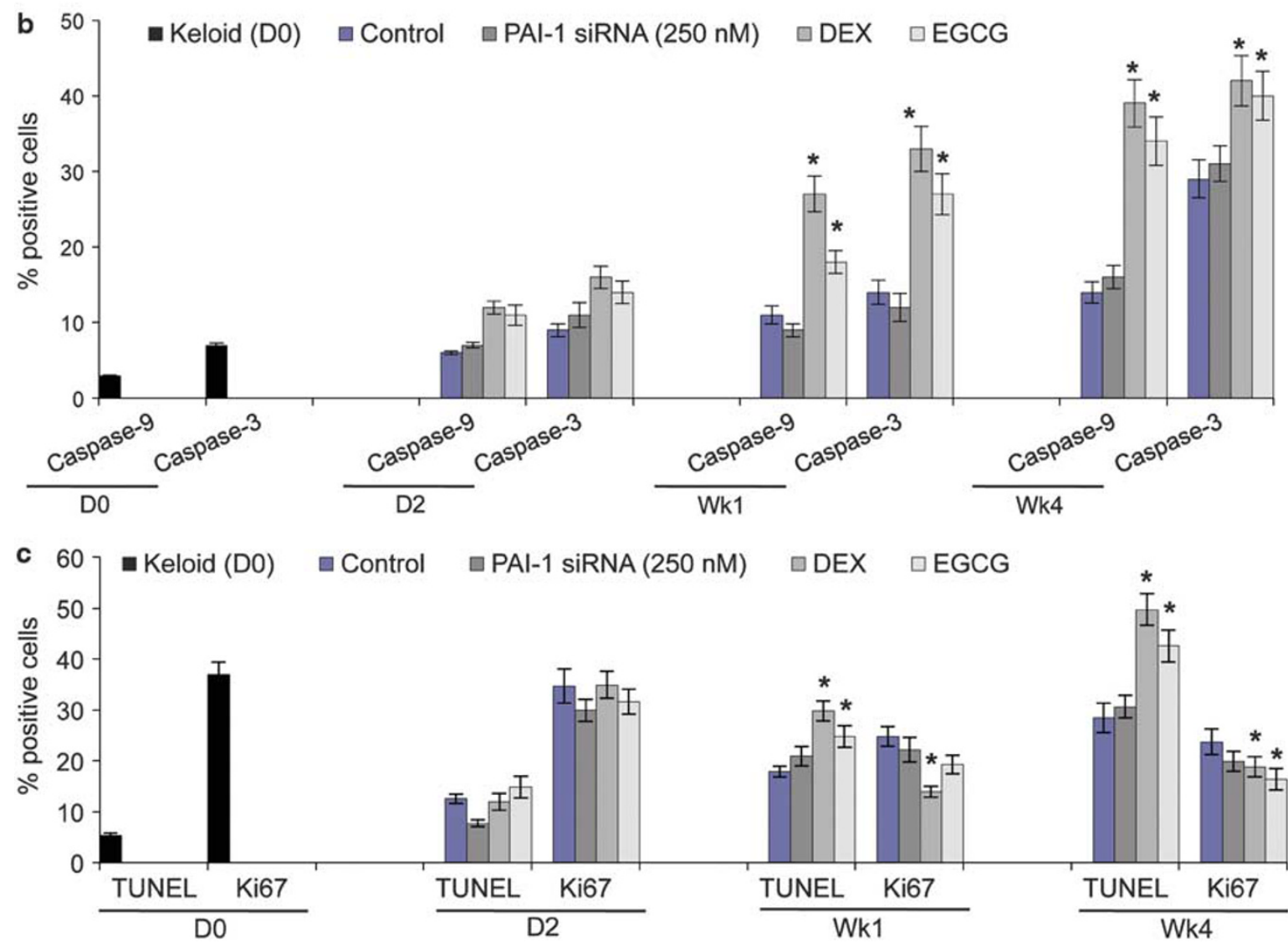

Figure 6 Effect of different treatments on apoptosis and proliferation of cells in the keloid organ culture (OC) model. (a) Immunofluorescence micrographs of caspase-9 (red) and -3 (green) after different treatments in keloid OC model. Micrographs were taken using confocal microscopy at $\times 400$ (yellow scale bar) and $\times 1000$ (white scale bar) magnifications. (b) Immunomorphometric quantification of caspase-9 and -3 expressions. ${ }^{*} P<0.05$ relative to vehicle-treated control. (c) Immunomorphometric analysis of terminal dUTP nick-end labeling (TUNEL) assay and cell proliferation using Ki-67 marker upon different treatments. Immunomorphometric analysis was generated from five random micrographs taken at $\times 400$ magnification, and $\%$ of positive cells was calculated. ${ }^{*} P<0.03$ relative to vehicle-treated control. Bars represent standard error. (Note: D0: day 0 , indicates in either normal skin or keloid scar, without any treatment.) E: epidermis;D: dermis; EGCG: ( - )-epigallocatechin-3-gallate. 


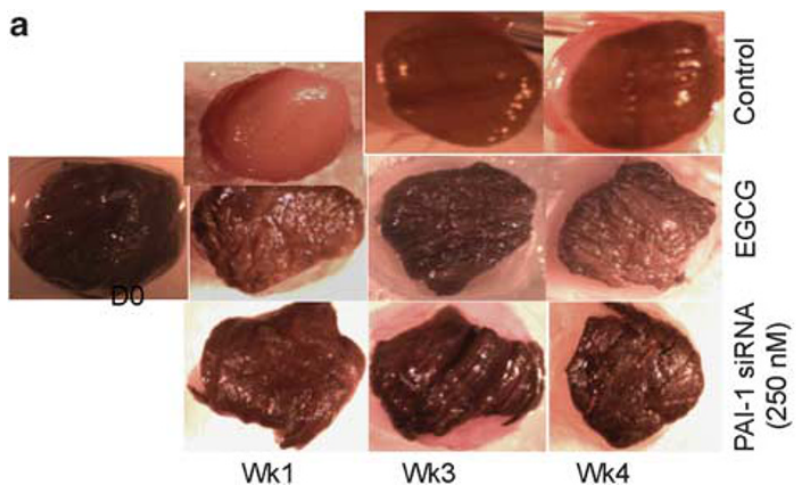

Figure 7 Shrinkage of the keloid organ culture (OC) after different treatments. (a) Representative photographs of keloid OC volume shrinkage with or without treatment captured at $\times 10$ magnification using stereo microscope (Leica, Milton Keynes, UK) using stereo microscope (Leica). (b) Average weights of the keloid OC after different treatments. ${ }^{*} P \leq 0.03$ relative to vehicle-treated control. EGCG: ( - )-epigallocatechin-3-gallate; PAI-l: plasminogen activator inhibitor-1; siRNA: small interfering RNA; D: day; Wk: week.

a

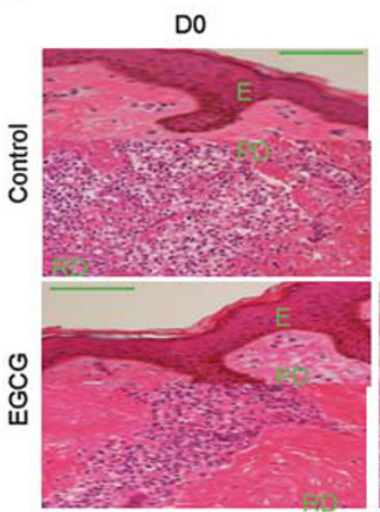

b

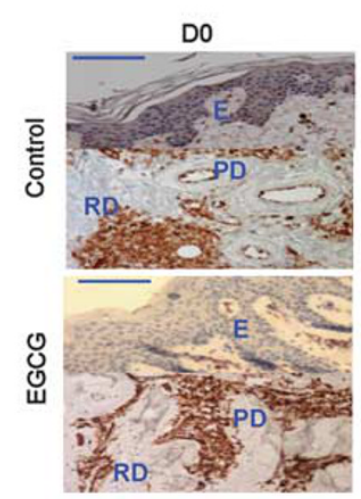

\section{Cellularity / Inflammation}
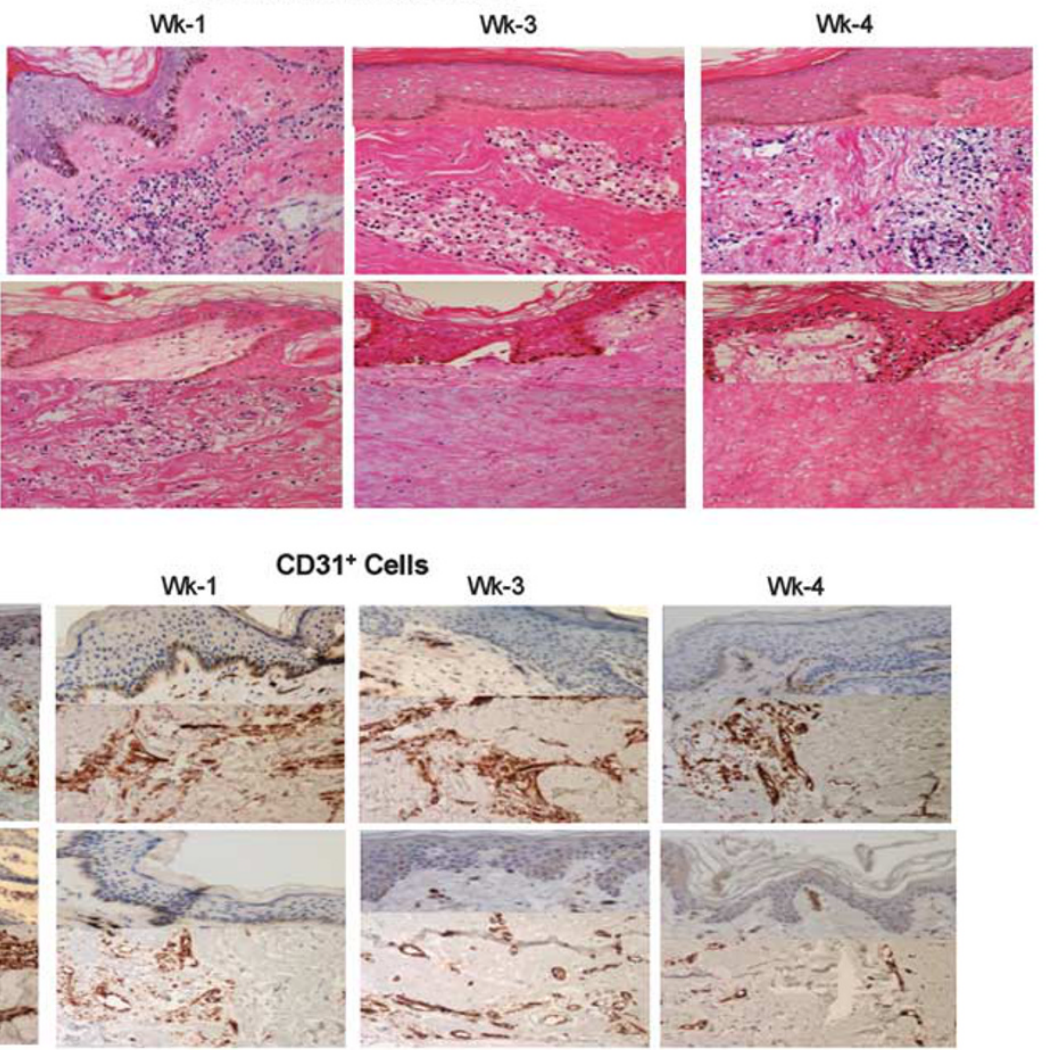

d
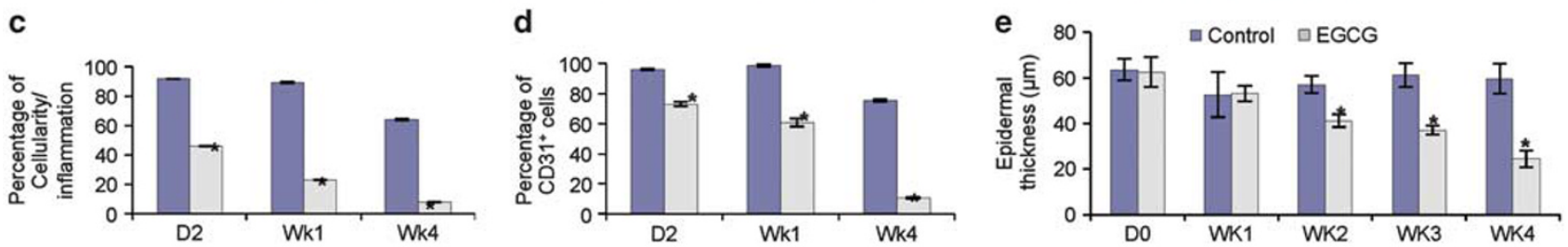

Figure 8 Effect on cellularity, blood vessels, and epidermal thickness after different treatments. (a) Hematoxylin and eosin (H\&E) and (b) CD31 immunoreactivity micrographs with and without ( - )-epigallocatechin-3-gallate (EGCG) treatment at different time points. Scale bar: $\times 200$ magnification. (c and d) Immunomorphometric quantitative analysis of cellularity (H\&E) and endothelial cells' (CD31 ${ }^{+}$cells') percentage after EGCG treatments generated from four random micrographs taken at $\times 200$ magnification. (e) Average epidermal shrinkage upon EGCG treatment in micrometers (measurement does not include stratum corneum). ${ }^{*} P<0.05$ relative to vehicle-treated control. Bars represent standard error. (Note: D0 indicates day 0 , in keloid scar without any treatment.) D: day, Wk: week, E: epidermis, PD: papillary dermis; RD: reticular dermis. 


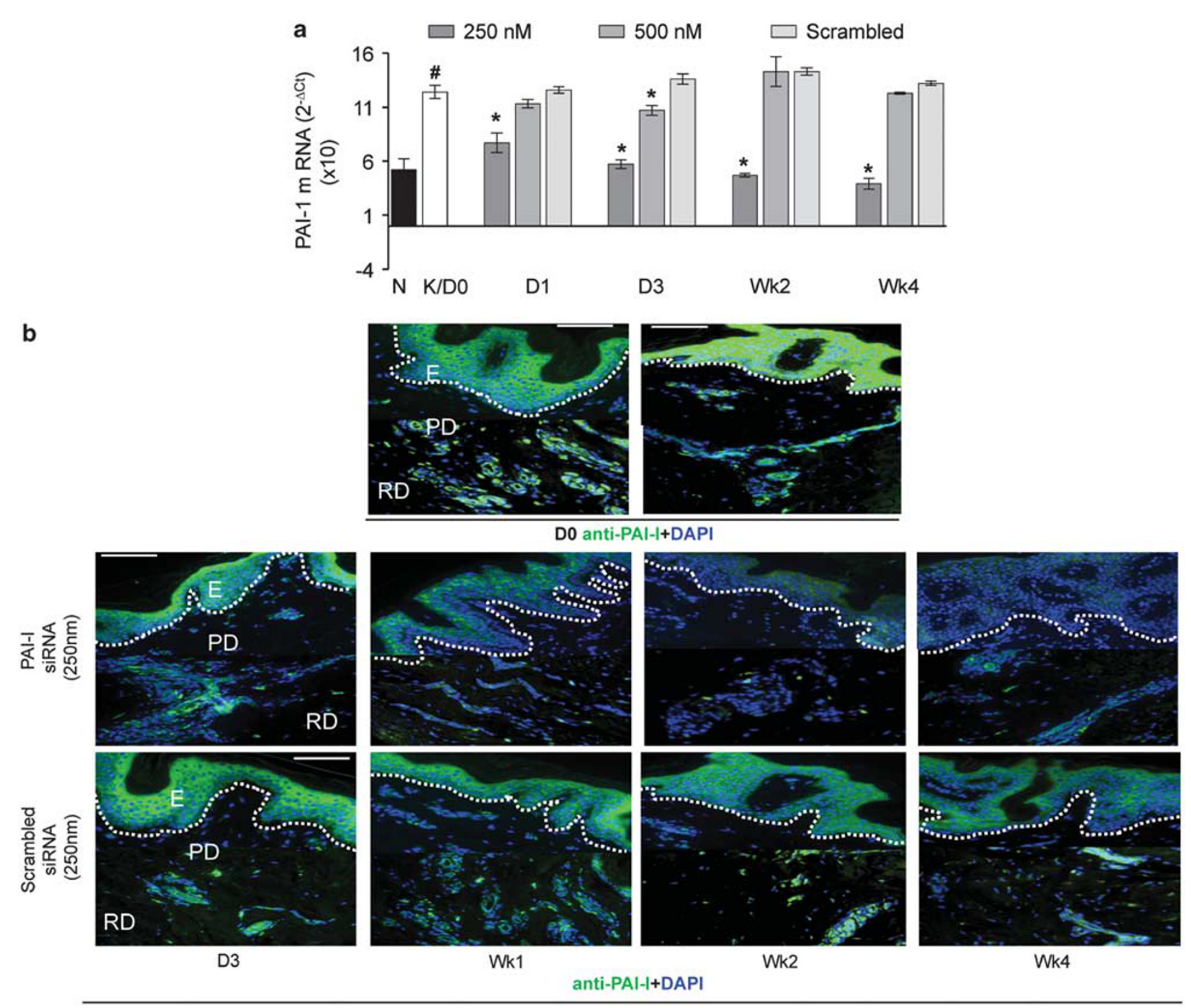

Figure 9 Successful demonstration of PAI-I: plasminogen activator inhibitor-1 (PAI-1) knockdown in keloid organ culture (OC) model using small interfering RNA (siRNA) technology. (a) PAI-1 mRNA and (b) PAl-1 protein expression profile after transfection of PAI-1 siRNA. " $P=0.01$ : relative to normal skin and ${ }^{*} P<0.05$ : relative to scrambled siRNA group. Bars represent standard error. (Note: D0: day 0 indicates either normal skin or keloid scar tissue/tissue transported media collected after surgery, without any treatment before the start of OC.) Scale bar: $\times 200$ magnification. D: day; Wk: week; K: keloid at day 0; N: normal skin at day 0; E: epidermis; PD: papillary dermis; RD: reticular dermis; DAPI: 4',6-diamidino-2-phenylindole.

the tissue analyses after PAI-1 siRNA treatment were conducted only with the $250 \mathrm{nM}$ PAI-1 siRNA-treated group.

Keloid shrinkage assay demonstrated a significant decrease of keloid volume by $28 \%$, after 4 weeks (Figures $7 \mathrm{a}$ and b). However, no obvious differences in comparison to control was observed in the caspase- 9 and -3 (Figures $6 a$ and b), TUNEL, and proliferation (Ki-67) (Figure 6c) analyses. Therefore, the keloid shrinkage induced by PAI-1 silencing appears to result primarily from its effects on collagen production in situ.

\section{DISCUSSION}

Here, we present a novel assay for the long-term, serum-free OC of native human keloid tumors within their natural tissue habitat. This explicitly includes keloid-associated epidermis, blood vessels, mast cells, and perilesional stroma, all of which have been implicated in the pathobiology of KD. Using a unique keloid OC model, ${ }^{6}$ we provide the first functional evidence that EGCG and PAI-1 silencing effectively inhibit growth and induce shrinkage of human keloid tissue in situ. Specifically, EGCG reduced keloid size, intrakeloid proliferation, collagen-I and -III production, mast cell numbers, blood vessel, and the expression of major profibrotic factors, while it increased intrakeloid apoptosis and induced epidermal shrinkage. PAI-1 knockdown in keloid OC, which represents the first successful gene silencing in intact human keloid tissue, also induced keloid tissue shrinkage, largely via its inhibitory effect on collagen 

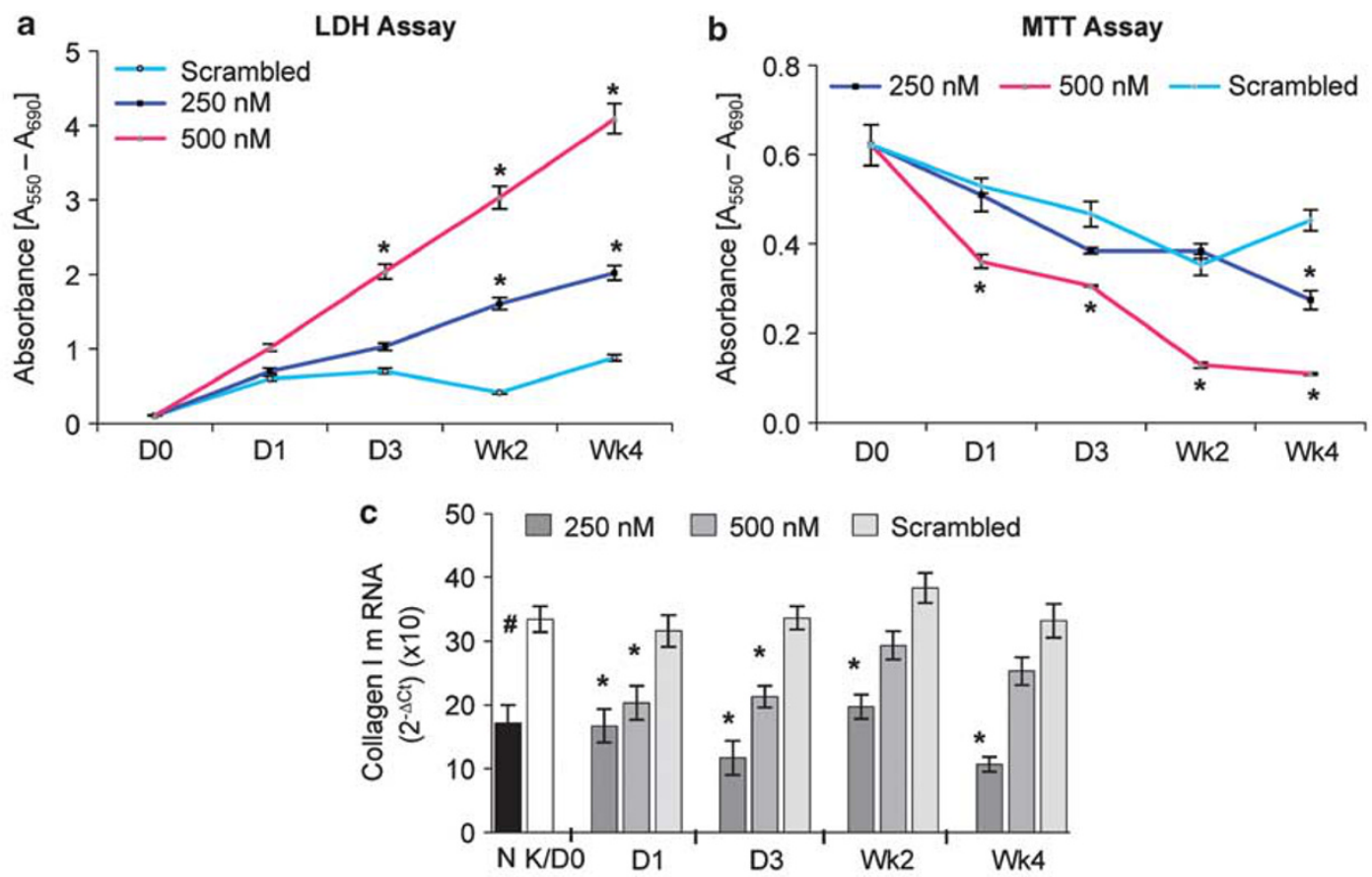

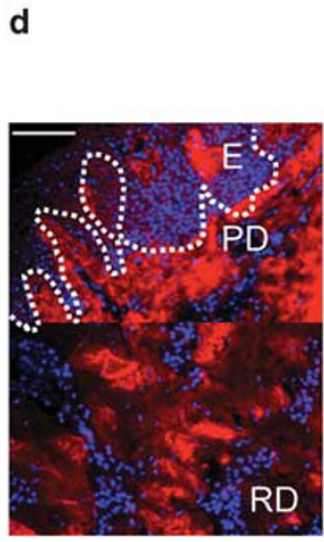

DO

anti-Collagen-1+DAPI
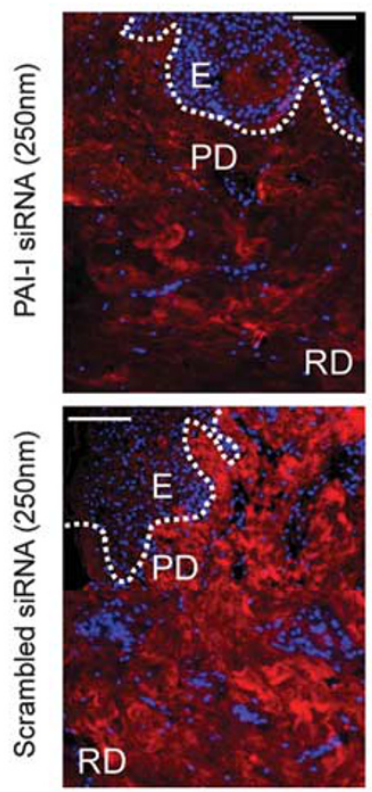

D3
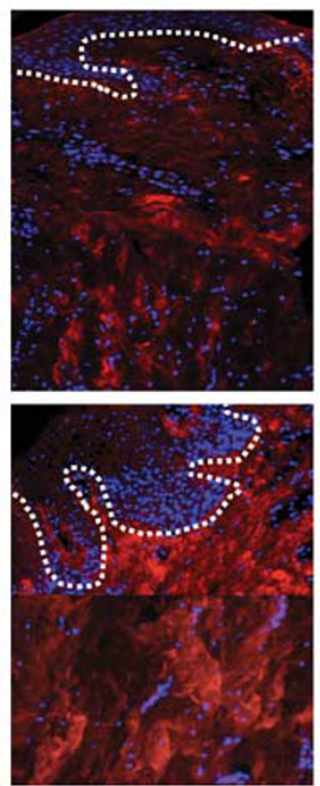

Wk1
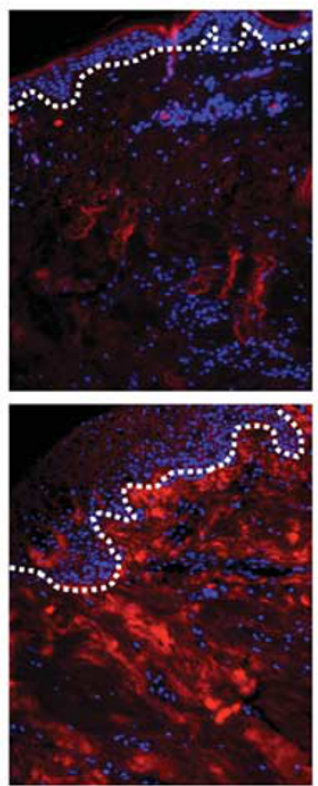

Wk2
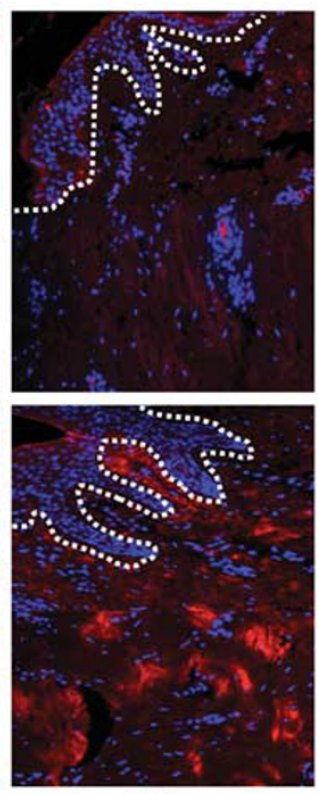

Wk4

anti-Collagen-1+DAPI

Figure 10 Efficiency and effect of plasminogen activator inhibitor-1 (PAI-1) short interfering RNA (siRNA) in the keloid organ culture (OC).

(a) Cytotoxic activity, (b) viability/metabolic activity, and (c) collagen-I mRNA expression upon PAI-1 knockdown using PAI-1 siRNA transfection. (d) Immunohistochemistry analysis for collagen-1 expression pattern in PAI-1 knockdown keloid OC tissue samples at different time points. Bars represent standard error. Scale bar: $\times 200$ magnification. (Note: D0: day 0, indicates keloid scar tissue, without any treatment before the start of OC). LDH: lactate dehydrogenase; MTT: methylthiazol tetrazolium; D: day; Wk: week; E: epidermis; PD: papillary dermis; RD: reticular dermis; DAPI: 4',6-diamidino-2-phenylindole.

synthesis. Therefore, it deserves systematic testing to establish whether application of EGCG and/or PAI-1 silencing before and after surgical excision can reduce size of $\mathrm{KD}$ as well as prevent its recurrence in combination with surgery.
In addition, this study shows that our recently reported assay system for the long-term, serum-free OC of native human keloid, within its natural tissue habitat, is optimally suited for pathophysiological/mechanistic keloid research that would otherwise only be possible in (non-existing) 

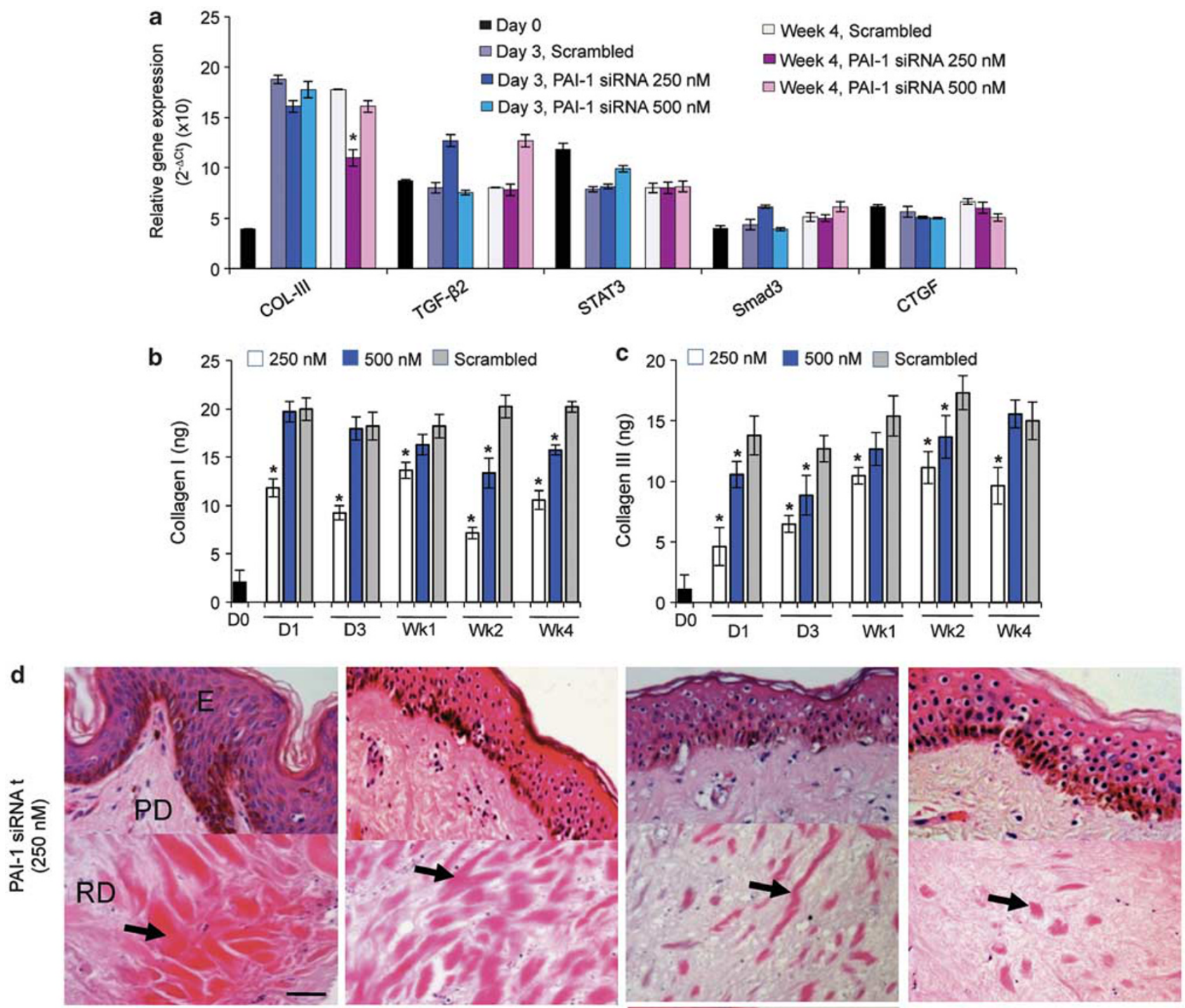

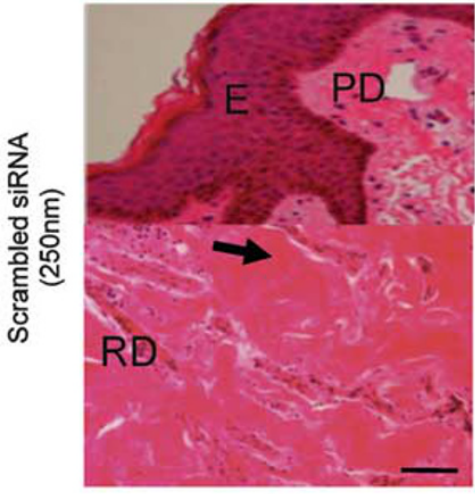

DO

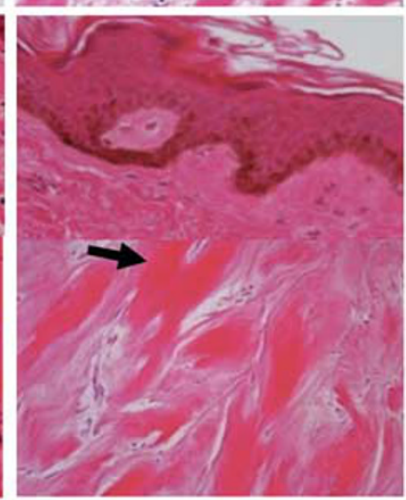

D3

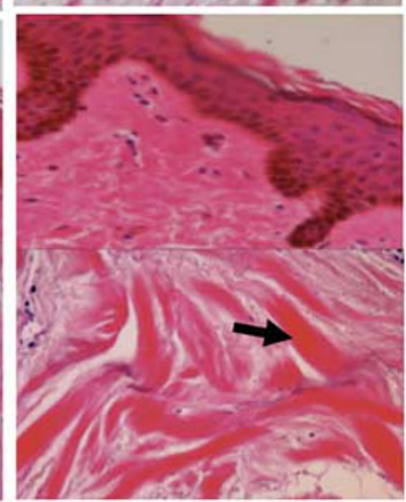

Wk2

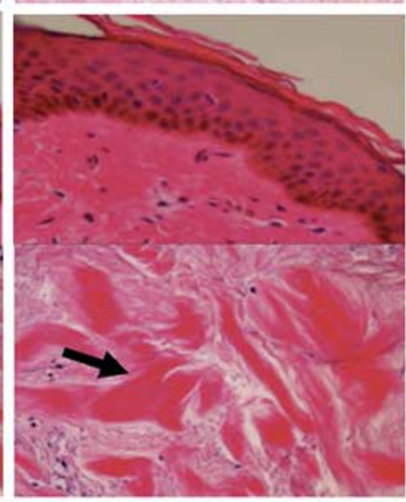

Wk4

Figure 11 Relative gene and protein expression of profibrotic molecules after plasminogen activator inhibitor-1 (PAl-1) silencing. (a) Relative gene expression of profibrotic molecules and (b) protein expression of collagen I and (c) collagen III after PAI-1 silencing treatment. ${ }^{*} P \leq 0.05$ comparing PAl-1 short interfering RNA (siRNA)-treated with scrambled control. Bars represent standard error (Note: D0: day 0, indicates either normal skin or keloid scar tissue/tissue-transported media collected after surgery, without any treatment before being organ cultured). (d) Hematoxylin and eosin micrographs of PAl-1-silenced keloid organ culture (OC). Scale bar: $\times 400$ magnification. Bars represent standard error. (Note: D0: day 0 indicates either normal skin or keloid scar tissue/tissue transported media collected after surgery, without any treatment before the start of OC). D: day; Wk: week; E: epidermis; PD: papillary dermis; RD: reticular dermis; COL, collagen; CTGF, connective tissue growth factor; STAT3: signal transducer and activator of transcription 3; TGF: tumor growth factor. 
transgenic animal model or keloid cell culture. ${ }^{6}$ Furthermore, this novel, clinically highly relevant assay enables direct assessment of innovative and promising antikeloid therapies at the preclinical level (compared with DEX as a positive control) before proceeding to the clinical trial stages.

Glucocorticoids are still the gold standard pharmacological therapy for the management of $\mathrm{KD}^{20}$ Thus, here we have used DEX as a positive control. DEX decreased both collagen-I and -III at mRNA levels; however, there was no significant effect at protein levels. The reason for this may be that collagen-I and -III mRNA were degraded by DEX, whereas there may not have been any effect at the protein level. DEX, however, induces apoptosis in many cell types and (on the basis of in vitro studies) is thought to induce keloid regression through suppression of VEGF and fibroblast proliferation. ${ }^{21}$ In addition, DEX inhibits MMPs in ocular fibroblasts. ${ }^{22}$ While DEX downregulated the expression of profibrotic pathways, including VEGF, TGF$\beta 2$ at mRNA level in ex vivo keloid OC system, DEX also induced CTGF overexpression.

This is in line with previous in vitro reports that had studied cultured murine fibroblast and hepatocytes..$^{23,24}$ Thus, DEX also exerts an undesirable effect by stimulating the gene and protein expression of a putative keloidpromoting CTGF, as elevated CTGF levels correlate with the increased matrix deposition, and formation of tissue fibrosis. ${ }^{18,19}$ Our findings may offer a reasonable explanation for the high recurrence rate of keloid after glucocorticoid treatment. ${ }^{1}$ Therefore, the current keloid OC model appears to be capable of detecting both desired and potentially undesirable effects of candidate antikeloid agents at the preclinical level. Here, we used $50 \mu \mathrm{g} / \mathrm{ml}$ DEX, which was derived from our previous in vitro work on fibroblasts using real-time cell analyzer on a microelectronic sensory array. ${ }^{25}$ Therefore, from this monoculture experiments, we extrapolated the optimal concentration for the keloid OC. Taken together, the prolonged administration of DEX provides a sensitive testing system for checking the effects of candidate antikeloid agents on the expression of important signaling pathways relevant to keloid pathobiology. Such an effect of DEX observed in the OC model also emphasizes that DEX deserves systematic exploration in future $\mathrm{KD}$ management.

The increased recurrence of keloid, despite the availability of different treatments, has driven research to identify and investigate novel therapeutic modalities. One of these narrative modalities is green tea extract, EGCG, which has been extensively studied as a therapeutic compound for several cancers. ${ }^{26}$ Recent work on EGCG in cell culture proposed a potential use of EGCG as an antikeloid treatment. ${ }^{4,10}$ Our OC data support the view that these prior observations with EGCG are of major clinical interest. In addition, we show that EGCG downregulates multiple profibrotic pathways (including PDGF, CTGF, MMPs (2 and 9), collagen, PI-3K, and STAT3). This is supported with the documented inhibitory effects of EGCG on tumour invasion, metastasis, and angiogenesis, both in vitro and in vivo. ${ }^{26}$ These effects observed here may limit the growth and possibly the recurrence of $\mathrm{KD}$. Our new keloid OC data suggest that intralesional injection of EGCG is an attractive candidate to limit the growth, and possibly the recurrence of $\mathrm{KD}$ in vivo. It has been shown that administration of EGCG in animal trials induced less side effect and is welltolerated at minimum doses depending on the route of administration $(500 \mathrm{mg} / \mathrm{kg}$ orally and $0.09 \%(1.8 \mathrm{mg} / \mathrm{kg})$ EGCG intradermally)..$^{27,28}$

The strong association of mast cells with fibrosis has been well established in several conditions, including KD. ${ }^{29}$ EGCG can attenuate mast cell-induced collagen production and fibroblast proliferation in keloid in vitro, coculture models through STAT3 and PI-3K pathways. ${ }^{10}$ That EGCG almost depleted mast cell numbers in the current keloid OC model confirms the potent impact of EGCG on mast cells and raises the possibility that the EGCG-induced reduction of intrakeloid collagen production may have occurred at least, in part, via downregulation of STAT3 and PI-3K.

Gene transfection using RNAi in human keloid cells either using cell culture or coculture was made possible with siRNA, ${ }^{12}$ as well as in organotypic normal skin model. ${ }^{30}$ However, there have been no studies to date investigating the applicability of RNAi to silence a target gene in a keloid OC model, to evaluate its efficiency as a potential antikeloid treatment in situ. Here, we demonstrate that satisfactory knockdown efficiency of PAI1 can be achieved even in keloid OC, suggesting that other keloid-promoting genes may also be silenced in situ and no doubt encourages further investigation into the effect of target gene knockdown both for obtaining better mechanistic insights into KD pathobiology in situ and for exploring novel candidate antikeloid therapies. PAI-1 siRNA was sustained for at least 5 days in our keloid OC model, as revealed by decreased PAI-1 mRNA expression (data not shown). Importantly, PAI-1 knockdown was efficient at a lower concentration $(250 \mathrm{nM})$ in OC model but a higher concentration $(500 \mathrm{nM})$ showed cytotoxicity and increased cell death. This may be due to offtarget effects, as an increased concentration of siRNA may compromise the specificity of the siRNA target. The use of RNAi technology in keloid OC may allow us to investigate the direct role of target genes in the epidermis/dermis without the difficulties imposed in an animal model, as animals do not develop KD.

The shrinkage of keloid OC and collagen deposition defined in this study indicates that the inhibitory effect of PAI-1 siRNA could be used as potential antikeloid treatment in the future. Such an inhibitory effect on collagen synthesis was also confirmed previously in an in vitro keloid model. ${ }^{12}$ Despite the link between PAI-1 and apoptosis, ${ }^{31}$ no significant changes were observed in our OC model. This could be explained by the low siRNA concentration used $(250 \mathrm{nM})$. Nonetheless, DEX appears to be more effective than EGCG and PAI-1 siRNA in its ability to induce 
apoptosis, and cytotoxicity in situ. On the other hand, reduction of collagen synthesis and shrinkage of keloid OC were more efficient with EGCG and PAI-1.

In the initial experiments at both the molecular and cellular level, we also compared proliferation rate and the expression pattern of fibrotic markers (without any treatment, on day 0 cells or tissue samples) in keloid $v s$ normal skin $v s$ extralesional tissue/primary fibroblasts (tissue collected away from the keloid lesion, from the same keloid patients, denoted as extralesional). Interestingly, we did not find a statistically significant difference in proliferation and in fibrotic markers expression pattern, between extralesional (same ethnic groups as keloid) vs normal skin primary fibroblasts, suggesting that extralesional tissue/primary fibroblasts behave similar to normal skin tissue/primary fibroblasts obtained from white ethnic group (our unpublished data). Hence, in this study, we excluded extralesional tissue, and compared the expression of collagen and PAI-1 between normal and keloid tissue at D0 (untreated only).

Given the unexpected, potentially undesired stimulatory effects of DEX on CTGF expression, which may be countered by EGCG, suggest that combination therapies that include EGCG and PAI-1 antagonists, alone or in conjunction with glucocorticoids, are promising novel antikeloid agents. These can potentially be used to reduce keloid volume before primary surgery to reduce keloid recurrence after excision. The clinical application of these potential compounds may involve the administration of EGCG or blockade of PAI topically and/or via an intralesional injection. The most ideal delivery format likely will be based on the successful penetration of the aforementioned topical administration into the dense keloid scar tissue. It is envisaged that for the larger lesions, a combination of surgical debulking as well as topical application or intralesional injection will be considered. The timing of combination therapy will be borne out by future clinical trials, evaluated by comparing topical and/or intralesional injection to keloid lesions pre- and postsurgical excision.

Supplementary Information accompanies the paper on the Laboratory Investigation website (http://www.laboratoryinvestigation.org)

\section{ACKNOWLEDGEMENTS}

We acknowledge study participants and nursing staff of South Manchester University Hospitals NHS Foundation Trust. We thank the GAT family foundation for their generous support of this work. $A B$ received a personal award from the NIHR (UK).

\section{DISCLOSURE/CONFLICT OF INTEREST}

The authors declare no conflict of interest.

1. Juckett G, Hartman-Adams H. Management of keloids and hypertrophic scars. Am Fam Phys 2009;80:253.

2. Shih B, Garside E, McGrouther DA, et al. Molecular dissection of abnormal wound healing processes resulting in keloid disease. Wound Rep Regen 2010;18:139-153.
3. Syed F, Ahmadi E, lqbal SA, et al. Fibroblasts from the growing margin of keloid scars produce higher levels of collagen I and III compared with intralesional and extralesional sites: clinical implications for lesional site-directed therapy. Br J Dermatol 2010;164:83-96.

4. Park G, Yoon BS, Moon J-H, et al. Green tea polyphenol epigallocatechin-3-gallate suppresses collagen production and proliferation in keloid fibroblasts via inhibition of the STAT3-signaling pathway. J Invest Dermatol 2008;128:2429-2441.

5. Kischer CW, Sheridan D, Pindur J. Use of nude (athymic) mice for the study of hypertrophic scars and keloids: vascular continuity between mouse and implants. Anat Rec 1989;225:189-196.

6. Bagabir R, Syed F, Paus R, et al. Long-term organ culture of keloid disease tissue. Exp Dermatol 2012;21:376-381.

7. Varani J, Perone P, Inman DR, et al. Human skin in organ culture. Elaboration of proteolytic enzymes in the presence and absence of exogenous growth factors. Am J Pathol 1995;146:210-217.

8. Lu Z, Hasse S, Bodo E, et al. Towards the development of a simplified long term organ culture method for human scalp skin and its appendages under serum free conditions. Exp Dermatol 2007;16: 37-44.

9. Duong HS, Zhang Q, Kobi A, et al. Assessment of morphological and immunohistological alterations in long-term keloid skin explants. Cells Tissues Organs 2005;181:89-102.

10. Zhang $\mathrm{Q}$, Kelly $\mathrm{AP}$, Wang $\mathrm{L}$, et al. Green tea extract and (-)epigallocatechin-3-gallate inhibit mast cell-stimulated type I collagen expression in keloid fibroblasts via blocking PI-3K/Akt signaling pathways. J Invest Dermatol 2006;126:2607-2613.

11. Tuan TLZJ, Sun B, Nichter LS, et al. Elevated levels of plasminogen activator inhibitor-1 may account for the altered fibrinolysis by keloid fibroblasts. J Invest Dermatol 1996;106:1007-1011.

12. Tuan $T L$, Hwu $P, H o w$, et al. Adenoviral overexpression and small interfering RNA suppression demonstrate that plasminogen activator inhibitor-1 produces elevated collagen accumulation in normal and keloid fibroblasts. Am J Pathol 2008;173:1311-1325.

13. Foitzik K, Krause K, Conrad F, et al. Human scalp hair follicles are both a target and a source of prolactin, which serves as an autocrine and/or paracrine promoter of apoptosis-driven hair follicle regression. Am J Pathol 2006;168:748-756.

14. Christoph T, Muller-Rover $\mathrm{S}$, Audring $\mathrm{H}$, et al. The human hair follicle immune system: cellular composition and immune privilege. $\mathrm{Br} J$ Dermatol 2000;142:862-873.

15. Syed F, Thomas A, Singh S, et al. In vitro study of novel collagenase (Xiaflex ${ }^{\circledR}$ ) on Dupuytren's disease fibroblasts displays unique drug related properties. PLoS One 2012;7:e31430.

16. Smith JC, Boone BE, Opalenik SR, et al. Gene profiling of keloid fibroblasts shows altered expression in multiple fibrosis-associated pathways. J Invest Dermatol 2007;128:1298-1310.

17. Peltonen J, Hsiao LL, Jaakkola $\mathrm{S}$, et al. Activation of collagen gene expression in keloids: co-localization of type I and VI collagen and transforming growth factor-1 mRNA. J Invest Dermatol 1991;97: 240-248.

18. Frazier KWS, Kothapalli $\mathrm{D}$, Klapper $\mathrm{H}$, et al. Stimulation of fibroblast cell growth, matrix production, and granulation tissue formation by connective tissue growth factor. J Invest Dermatol 1996;107: 404-411.

19. Igarashi A, Nashiro K, Kikuchi K, et al. Connective tissue growth factor gene expression in tissue sections from localized scleroderma, keloid, and other fibrotic skin disorders. J Invest Dermatol 1996;106: 729-733.

20. Gadson PF, Russell JD, Russell SB. Glucocorticoid receptors in human fibroblasts derived from normal dermis and keloid tissue. J Biol Chem 1984;259:11236-11241.

21. Wu W-S, Wang F-S, Yang KD, et al. Dexamethasone induction of keloid regression through effective suppression of VEGF expression and keloid fibroblast proliferation. J Invest Dermatol 2006;126: 1264-1271.

22. Saadat F, Raji A, Zomorodian $\mathrm{K}$, et al. Alteration in matrix metalloproteinases (MMPs) activity in fibroblast cell line by dexamethasone: a possible mechanism in corticosteroid-induced glaucoma. Iran J Allergy Asthma Immunol 2003;2:145-148.

23. Dammeier J, Beer H-D, Brauchle $M$, et al. Dexamethasone is a novel potent inducer of connective tissue growth factor expression. J Biol Chem 1998;273:18185-18190. 
24. Wickert L, Chatain N, Kruschinsky K, et al. Glucocorticoids activate TGFbeta induced PAI-1 and CTGF expression in rat hepatocytes. Comp Hepatol 2007;6:5.

25. Syed F, Bayat A. Superior effect of combination vs. single steroid therapy in keloid disease: A comparative in vitro analysis of glucocorticoids. Wound Rep Regen 2012;21:88-102.

26. Khan N, Mukhtar H. Cancer and metastasis: prevention and treatment by green tea. Cancer Metasts Rev 2010;29:435-445.

27. Isbrucker RA, Bausch J, Edwards JA, et al. Safety studies on epigallocatechin gallate (EGCG) preparations. Part 1: Genotoxicity. Food Chem Toxicol 2006;44:626-635.
28. Isbrucker RA, Edwards JA, Wolz E, et al. Safety studies on epigallocatechin gallate (EGCG) preparations. Part 2: Dermal, acute and shortterm toxicity studies. Food Chem Toxicol 2006;44:636-650.

29. Cohen IK, Beaven MA, Horakova Z, et al. Histamine and collagen synthesis in keloid and hypertrophic scar. Plast Reconstruct Surg 1973; 51:709.

30. Mildner M, Ballaun C, Stichenwirth M, et al. Gene silencing in a human organotypic skin model. Biochem Biophys Res Commun 2006;348: 76-82.

31. Balsara RD, Ploplis VA. Plasminogen activator inhibitor-1: the doubleedged sword in apoptosis. Thromb Haemost 2008;100:1029-1036. 